\title{
6-Acetonyl-N-methyl-dihydrodecarine, a New Alkaloid from Zanthoxylum riedelianum
}

\section{Carromberth C. Fernandes, ${ }^{a}$ Paulo C. Vieira,${ }^{b}$ Virgínia C. da Silva, ${ }^{c}$ Evandro L. Dall'Oglio, ${ }^{c}$ Luiz E. da Silva and Paulo T. de Sousa Jr. ${ }^{*}, c$}

\author{
${ }^{a}$ Departamento de Ciências da Natureza, Universidade Federal do Acre, 66915-900 Rio Branco-AC, Brazil \\ ${ }^{b}$ Departamento de Química, Centro de Ciências Exatas e de Tecnologia, Universidade Federal de São Carlos, \\ 13565-905 São Carlos-SP, Brazil

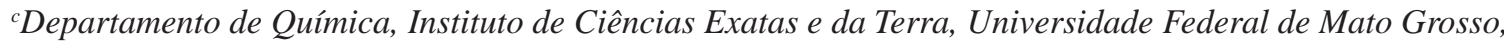 \\ 78060-900 Cuiabá- MT, Brazil
}

\begin{abstract}
Um novo alcalóide benzofenantridínico, 6-acetonil- $N$-metil-diidrodecarina foi isolado das raízes de Zanthoxylum riedelianum juntamente com lupeol, 6-acetonildiidroqueleritrina e 6-acetonildiidroavicina. As estruturas dessas substâncias foram estabelecidas com base na análise dos dados espectrométricos de IV, EM e RMN incluindo experimentos 2D.

A new benzophenanthridine alkaloid, 6-acetonyl- $N$-methyl-dihydrodecarine was isolated from Zanthoxylum riedelianum roots together with lupeol, 6-acetonyldihydrochelerythrine and 6-acetonyldihydroavicine. The structures were established from the IR, MS and NMR spectral data, including 2D-NMR experiments.
\end{abstract}

Keywords: Zanthoxylum riedelianum, Rutaceae, benzophenanthridine alkaloids

\section{Introduction}

The Zanthoxylum genus (Rutaceae) is composed by more than 200 species and largely distributed around the world. ${ }^{1}$ Chemically, this genus is characterized by alkaloids, ${ }^{2-7}$ cumarins, ${ }^{5,6,8}$ lignans, ${ }^{4,9,10}$ amides ${ }^{11,12}$ and terpenes. ${ }^{5,6,13,14}$ Ongoing studies have shown that Zanthoxylum exhibit a range of biological activities such as antichagas, ${ }^{2}$ tripanocidal, ${ }^{9}$ antiplasmodial, ${ }^{7}$ anti-HIV ${ }^{13}$ and antiinflammatory, ${ }^{8,10}$ as well as antihelmintic. ${ }^{12} \mathrm{Z}$. riedelianum is used in folk medicine as a decoction against different types of inflammations, rheumatism and skin stains. ${ }^{15}$ Previous works reported the identification of terpenes from the essential oil ${ }^{14}$ and lignans from the leaves and the stem bark. ${ }^{10}$ In this work we report the isolation and structural elucidation of a novel benzophenanthridine alkaloid, namely, 6-acetonyl- $N$-methyl-dihydrodecarine (1), together with two known alkaloids 6-acetonyldihydrochelerythrine (2) and 6-acetonyldihydroavicine (3) from the roots of $Z$. riedelianum.

\footnotetext{
*e-mail: teixeira@ufmt.br
}

\section{Experimental}

\section{General procedures}

Melting points were uncorrected. IR spectra were recorded on FTIR-Bomem-MB/100 model spectrophotometer using $\mathrm{NaCl}$ film. NMR spectra in $\mathrm{CDCl}_{3}$, were recorded on Bruker ARX-400 (400 MHz for ${ }^{1} \mathrm{H}$ and $100 \mathrm{MHz}$ for ${ }^{13} \mathrm{C}$ ); Bruker AC$200\left(200 \mathrm{MHz}\right.$ for ${ }^{1} \mathrm{H}$ and $50 \mathrm{MHZ}$ for ${ }^{13} \mathrm{C}$ ) and Varian-Mercury $300\left(300 \mathrm{MHz}\right.$ for ${ }^{1} \mathrm{H}$ and $75 \mathrm{MHz}$ for ${ }^{13} \mathrm{C}$ ) spectrometers, using tetramethylsilane (TMS) as internal standard. Electron Ionization Mass Spectra (ESI-MS) was undertaken employing a Quatro LC-Micromass UK model spectrometer. Optical rotations were determined on a Perkin-Elmer 341 polarimeter. CC: silica gel (Merck 70-230 mesh ASTM); TLC: silica gel $\mathrm{G} 60$ and silica gel $60 \mathrm{PF}_{254}$ (Merck) were used to analyze the fractions collected from column chromatography (CC) with visualization by UV (254 and $366 \mathrm{~nm}$ ), Dragendorff's reagent and exposure to iodine vapor.

\section{Plant material}

Zanthoxylum riedelianum (Engl.) was collected in Rio Manso Highway, km 22, Chapada dos Guimarães, Mato 
<smiles>[R]Oc1ccc2c(c1OC)[C@H](C(C)=O)N(C)c1c-2ccc2cc3c(cc12)OCO3</smiles>

Grosso State, Brazil. A voucher specimen (No. 24.080) was deposited at Universidade Federal de Mato Grosso Central Herbarium.

\section{Extraction and isolation}

Dried roots $(3.0 \mathrm{~kg})$ were powdered and extracted with hexane and methanol by maceration at room temperature. The macerates were concentrated under reduced pressure to yield the extracts A (21.5 g) and B (200.5 g) from hexane and methanol, respectively. The extract A was partitioned with hexane, dichloromethane, ethyl acetate and methanol. Solvents were removed under reduced pressure and the dichloromethane residue $(8.2 \mathrm{~g})$ was submitted to column chromatography, carried out in a gradient system from hexane, dichloromethane, ethyl acetate, acetone and methanol as mobile phase. The 174 collected fractions were reunited in 30 fractions. Fraction $10(1.0 \mathrm{~g})$ afforded the triterpene lupeol (170.0 mg, mp 162.5-164.2 ${ }^{\circ} \mathrm{C}$ ). Fraction 25 (200.0 mg) was submitted to preparative TLC with dichloromethane-methanol (2:8), affording the alkaloid 6-acetonyldihydrochelerythrine (2, 30.0 mg, mp 171.6-173.0 $\left.{ }^{\circ} \mathrm{C}\right)$.

The extract B was partitioned successively with hexane, dichloromethane, ethyl acetate and methanol. The dichloromethane residue $(530.0 \mathrm{mg})$ was submitted to column chromatography performed in a gradient system with hexane, dichloromethane, acetone and methanol as mobile phase. The 243 collected fractions were reunited in 33 fractions after TLC comparison. Fraction 4 (190.0 mg) was submitted to preparative TLC eluting with dichloromethane-methanol (1:9), affording the alkaloids 6-acetonyl- $N$-methyl-dihydrodecarine $(\mathbf{1}, 60.0 \mathrm{mg}, \mathrm{mp}$ 186-188 $\left.{ }^{\circ} \mathrm{C}\right)$ and 6-acetonyldihydroavicine $(3,57.0 \mathrm{mg}$, mp $\left.184-185^{\circ} \mathrm{C}\right)$.

\section{6-Acetonyl-N-methyl-dihydrodecarine, (1)}

Brown amorphous solid. $[\alpha]_{\mathrm{D}}^{21.5^{\circ}}-5.625\left(\mathrm{CHCl}_{3}\right.$; conc. $\left.0.014 \mathrm{~g} \mathrm{~mL}^{-1}\right)$. IR (NaCl film) $v_{\max } / \mathrm{cm}^{-1}: 3396,1708,1615$,<smiles>CC(=O)C[C@H]1c2cc3c(cc2-c2ccc4cc5c(cc4c2N1C)OCO5)OCO3</smiles>

3

1610, 1516, 1425, 1296, 1239. ${ }^{1} \mathrm{H} \mathrm{NMR}\left(\mathrm{CDCl}_{3}, 400 \mathrm{MHz}\right)$ and ${ }^{13} \mathrm{C} \mathrm{NMR}\left(\mathrm{CDCl}_{3}, 100 \mathrm{MHz}\right)$ (Table 1). ESIMS/MS: $\mathrm{m} / \mathrm{z}$ (rel. int.) $391[\mathrm{M}+\mathrm{H}]^{+}$.

Table 1. ${ }^{1} \mathrm{H}(400 \mathrm{MHz})$ and ${ }^{13} \mathrm{C}-\mathrm{NMR}(100 \mathrm{MHz})$ spectral data of $\mathbf{1}$ and 3, in $\mathrm{CDCl}_{3}$

\begin{tabular}{|c|c|c|c|c|}
\hline \multirow[t]{2}{*}{$\mathrm{C}$} & \multicolumn{2}{|r|}{1} & \multicolumn{2}{|r|}{3} \\
\hline & $\delta_{\mathrm{C}}$ & $\delta_{\mathrm{H}}(m, J$ in $\mathrm{Hz})$ & $\delta_{\mathrm{C}}$ & $\delta_{\mathrm{H}}(m, J$ in $\mathrm{Hz})$ \\
\hline 1 & 104.4 & $7.10(\mathrm{~s})$ & 107.7 & $6.82(\mathrm{~s})$ \\
\hline 2 & 148.5 & & 148.2 & \\
\hline 3 & 147.5 & & $147.6^{*}$ & \\
\hline 4 & 100.5 & $7.51(\mathrm{~s})$ & 103.6 & $7.29(\mathrm{~s})$ \\
\hline $4 a$ & 123.9 & & 124.8 & \\
\hline $4 \mathrm{~b}$ & 138.8 & & 131.0 & \\
\hline 6 & 54.8 & $5.0(\mathrm{dd}, 3.5,10.9)$ & 60.2 & $4.54(\mathrm{dd}, 5.7,8.7)$ \\
\hline $6 a$ & 123.3 & & 123.5 & \\
\hline 7 & 151.3 & & 100.5 & $7.54(\mathrm{~s})$ \\
\hline 8 & 144.9 & & 147.2 & \\
\hline 9 & 115.1 & $7.01(\mathrm{~d}, 8.4)$ & $147.6^{*}$ & \\
\hline 10 & 119.7 & $7.54(\mathrm{~d}, 8.4)$ & 104.3 & $7.11(\mathrm{~s})$ \\
\hline $10 \mathrm{a}$ & 131.0 & & 123.9 & \\
\hline $10 \mathrm{~b}$ & 127.5 & & 127.1 & \\
\hline 11 & 120.0 & $7.72(\mathrm{~d}, 8.5)$ & 119.8 & $7.64(\mathrm{~d}, 8.5)$ \\
\hline 12 & 124.7 & $7.51(\mathrm{~d}, 8.5)$ & 124.8 & $7.51(\mathrm{~d}, 8.5)$ \\
\hline $12 \mathrm{a}$ & 127.2 & & 128.3 & \\
\hline 1' $\left(-\mathrm{CH}_{2} \mathrm{CO}\right)$ & 46.5 & $\begin{array}{c}2.25(\mathrm{dd}, 3.5,15.2) \\
\text { and } 2.66(\mathrm{dd}, 10.9, \\
15.2)\end{array}$ & 48.2 & $\begin{array}{c}2.33(\mathrm{dd}, 5.7,15.7) \\
\text { and } 2.60(\mathrm{dd}, 8.7, \\
15.7)\end{array}$ \\
\hline $2^{\prime}\left(-\mathrm{COCH}_{3}\right)$ & 207.6 & & 207.7 & \\
\hline $3^{\prime}\left(-\mathrm{COCH}_{3}\right)$ & 31.5 & $2.02(\mathrm{~s})$ & 31.4 & $1.97(\mathrm{~s})$ \\
\hline $\mathrm{OCH}_{2} \mathrm{O}$ & 101.1 & $6.05(\mathrm{~s})$ & $\begin{array}{c}101.1 \\
\text { and } \\
101.9\end{array}$ & $\begin{array}{l}6.10(\mathrm{~d}, 2.5) \text { and } \\
\quad 6.0(\mathrm{~d}, 2.5)\end{array}$ \\
\hline $7-\mathrm{OCH}_{3}$ & 61.9 & $3.95(\mathrm{~s})$ & & \\
\hline 8-OH & & $5.30(\mathrm{~s})$ & & \\
\hline $\mathrm{N}-\mathrm{CH}_{3}$ & 42.4 & $2.65(\mathrm{~s})$ & 42.2 & $2.60(\mathrm{~s})$ \\
\hline
\end{tabular}

"Signals overlapped. 


\section{Results and Discussion}

The well known natural compounds lupeol, ${ }^{16}$ 6-acetonyldihydrochelerythrine (2) ${ }^{17}$ and 6-acetonyldihydroavicine $(3)^{18}$ were identified mainly by ${ }^{1} \mathrm{H}$ and ${ }^{13} \mathrm{C}$ NMR spectral analyses, comparing with previous literature data. ${ }^{16-18}$

Compound $\mathbf{1}$ presented a positive test with Dragendorff's reagent, indicating it to be an alkaloid. The IR spectrum presented bands at $3396\left(v_{\mathrm{OH}}\right), 1708\left(\mathrm{v}_{\mathrm{C}=\mathrm{O}}\right)$ 1615, 1516 $\mathrm{cm}^{-1}$ attributed to aromatic rings. The positive ESImass spectrum of $\mathbf{1}$ showed a quasi-molecular ion at $\mathrm{m} / \mathrm{z} 391[\mathrm{M}+\mathrm{H}]^{+}$pointing out to a molecular formula $\mathrm{C}_{23} \mathrm{H}_{21} \mathrm{NO}_{5}$. The ${ }^{1} \mathrm{H}$ NMR spectrum of $\mathbf{1}$, exhibited signals corresponding to six aromatic hydrogen, characteristic of a benzophenanthridine system. ${ }^{18}$ Accordingly, the aromatic region from compound (1) ${ }^{1} \mathrm{H}$ NMR spectrum exhibited signals of two pairs of one-hydrogen doublets $\left(\delta_{\mathrm{H}} 7.01(\mathrm{H}-9)\right.$ and $7.54(\mathrm{H}-10) ; 7.72(\mathrm{H}-11)$ and $7.51(\mathrm{H}-12))$ and two one-hydrogen singlets $\left(\delta_{\mathrm{H}} 7.10\right.$ $(\mathrm{H}-1)$ and $7.51(\mathrm{H}-4))$ indicating the presence of four aromatic hydrogens in ortho position and two isolated hydrogens. The ${ }^{1} \mathrm{H}$ NMR spectrum also displayed signals of methylenedioxide group at $\delta_{\mathrm{H}} 6.05(2 \mathrm{H}, \mathrm{AB}$ system $)$, hydroxyl group at $\delta_{\mathrm{H}} 5.30(\mathrm{~s}, 1 \mathrm{H})$, a methoxyl group at $\delta_{\mathrm{H}} 3.95(\mathrm{~s}, 3 \mathrm{H})$ and the $N$-methyl group at $\delta_{\mathrm{H}} 2.65(\mathrm{~s}, 3 \mathrm{H})$. In addition to the benzophenanthridine signals, the presence of an acetonyl group at C- 6 was indicated by a methyl singlet at $\delta_{\mathrm{H}} 2.02$ and the AMX system with $\delta_{\mathrm{H}}$ $2.25(J 3.5$ and $15.2 \mathrm{~Hz})$ and $2.66(J 10.9$ and $15.2 \mathrm{~Hz})$, respectively, and $\delta_{\mathrm{H}} 5.0(\mathrm{~J} 3.5$ and $10.9 \mathrm{~Hz})$, corresponding to the coupling constants between H-6 and the acetonyl methylene hydrogens in the ${ }^{1} \mathrm{H}$ NMR and ${ }^{1} \mathrm{H},{ }^{1} \mathrm{H}-\mathrm{COSY}$ spectra. The ${ }^{13} \mathrm{C}$ NMR spectrum also confirmed the acetonyl moiety with signals at $\left.207.6(\mathrm{C}=\mathrm{O}), 31.5\left(\mathrm{CO}_{\mathrm{CH}}\right)_{3}\right), 46.5$ $\left(-\mathrm{CH}_{2} \mathrm{CO}\right)$. Through the chemical shifts observed in the ${ }^{13} \mathrm{C},{ }^{1} \mathrm{H}-\mathrm{gHSQC}$ spectrum, it was possible the attribution of each carbon and its respective hydrogen (Table 1). The cross peaks observed in the HMBC spectrum showed longrange couplings from $\mathrm{H}-6\left(\delta_{\mathrm{H}} 5.0\right)$ and $\mathrm{H}-3$ ' $\left(\delta_{\mathrm{H}} 2.02\right)$ with C-2' $\left(\delta_{\mathrm{C}} 207.6\right)$, confirming the connection of the acetonyl moiety with C-6. Further correlations were observed between $\mathrm{OCH}_{3}\left(\delta_{\mathrm{H}} 3.95\right)$ with $\mathrm{C}-7\left(\delta_{\mathrm{C}} 151.3\right)$, as well as $\mathrm{H}-9$ $\left(\delta_{\mathrm{H}} 7.01\right)$ and $\mathrm{H}-10\left(\delta_{\mathrm{H}} 7.54\right)$ with $\mathrm{C}-8\left(\delta_{\mathrm{C}} 144.9\right)$ and $\mathrm{C}-10$ $\left(\delta_{\mathrm{C}} 119.7\right)$, indicating that position 8 is substituted; finally, $N-\mathrm{CH}_{3}\left(\delta_{\mathrm{H}} 2.65\right)$ with C-6 $\left(\delta_{\mathrm{C}} 54.8\right)$. The absence of the methoxyl group in C-7 moves away the effect of the oxygen atom in C-6, justifying its largest chemical shift $\left(\delta_{C} 60.2\right)$ in compound $\mathbf{3}$, when compared with the correspondent $\delta_{\mathrm{C}} 54.8$, attributed to C-6 in compound $\mathbf{1}$; thus revealing $\Delta_{\mathrm{C}}=54.9-60.2=-5.3 \mathrm{ppm}$ as $\gamma$ effect. Therefore, the structure of 1 was established as 6-acetonyl- $N$-methyldihydrodecarine, a decarine derivative. ${ }^{18}$ Previous publications, ${ }^{17,18}$ however, reported the isolation of the unstable parent alkaloids avicine and nitidine. Although acetone derivatives of avicine and nitidine have been reported in the literature, ${ }^{19-21}$ as well as acetone aducts of two other benzophenantridine alkaloids, ${ }^{22,23}$ it is controversial, however, whether those acetone derivatives are really present on the plant extract or were isolated as artifacts, due to the greater stability of the acetone aducts as compared to the parent alkaloids avicine and nitidine.

\section{Acknowledgments}

The authors are grateful to CNPq, FAPEMAT and Centro de Pesquisa do Pantanal (CPP) for scholarships and financial support, as well as to Dr. A. Gilberto Ferreira, UFSCar, for the $400 \mathrm{MHz}$ NMR spectra.

\section{Supplementary Information}

Supplementary data of alkaloids structures as 1 and $2 \mathrm{D}{ }^{1} \mathrm{H}$ and ${ }^{13} \mathrm{C}$ NMR spectra are available free of charge at http://jbcs.sbq.org.br, as PDF file.

\section{References}

1. Talapatra, S. K.; Dutta, S. K.; Talapatra, B.; Phytochemistry 1973, 12, 729.

2. Ferreira, M. E.; Nakayama, H.; Arias, A. R.; Schinini, A.; de Bilbao, N. V.; Serna, E.; Lagoutte, D.; Soriano-Agatón, F.; Poupon, E.; Hocquemiller, R.; Fournet, A.; J. Ethnopharmacol. 2007, 109, 258.

3. Hu, J.; Zhang, W. D.; Shen, Y. H.; Zhang, C.; Xu, L.; Liu, R. H.; Wang, B.; Xu, X. K.; Biochem. Syst. Ecol. 2007, 35, 114.

4. Rahman, M. M.; Islam, M. A.; Khondkar, P.; Gray, A. I.; Biochem. Syst. Ecol. 2005, 33, 91.

5. Arruda, M. S. P.; Fernandes, J. B.; Vieira, P. C.; da Silva, M. F. G. F.; Pirani, J. R.; Biochem. Syst. Ecol. 1992, 20, 173.

6. Oliveira, E. L.; Freitas, P. C.; Guedes, M. L. S.; Velozo, E. S.; Rev. Bras. Farmacogn. 2002, 12, 29.

7. Jullian, V.; Bourdy, G.; Georges, S.; Maurel, S.; Sauvain, M.; J. Ethnopharmacol. 2006, 106, 348.

8. Chen, I. S.; Lin, Y. C.; Tsai, I. L.; Teng, C. M.; Ko, F. N.; Ishikawa, T.; Ishii, H.; Phytochemistry 1995, 39, 1091.

9. Bastos, J. K.; Albuquerque, S.; Silva, M. L. A.; Planta Med. 1999, 65, 541.

10. Lima, L. M.; Perazzo, F. F.; Carvalho, J. C. T.; Bastos, J. K.; J. Pharm. Pharmacol. 2007, 59, 1151.

11. Chen, I. S.; Chen, T. L.; Lin, W. Y.; Tsai, I. L.; Chen, Y. C.; Phytochemistry 1999, 52, 357. 
12. Navarrete, A.; Hong, E.; Planta Med. 1996, 62, 250.

13. Cheng, M. J.; Lee, K. H.; Tsai, I. L.; Chen, I. S.; Bioorg. Med. Chem. 2005, 13, 5915.

14. Guy, I.; Charles, B.; Guinaudeau, H.; Ferreira, M. E.; Arias, A. R.; Fournet, A.; Pharm. Biol. 2001, 39, 152.

15. Guarim Neto, G.; Plantas Utilizadas na Medicina Popular do Estado de Mato Grosso, Associação Brasileira de Educação Agrícola Superior: Brasília, 1996.

16. Mahato, S. B.; Kundu, A. P.; Phytochemistry 1994, 37, 1517.

17. Julián, A.; Delgado, G.; Rev. Soc. Quim. Mex. 2001, 45, 189.

18. Nissanka, A. P. K.; Karunaratne, V.; Bandara, B. M. R.; Kumar, V.; Nakanishi, T.; Nishi, M.; Inada, A.; Tillekeratne, L. M. V.; Wijesundara, D. S. A.; Gunatilaka, A. A. L.; Phytochemistry 2001, 56, 857.
19. Arthur, H. R., Hui, W. H.; Ng, Y. L.; J. Chem. Soc. 1959, 4007.

20. Gopinath, K. W.; Kohli, J. M.; Kahn, M. S. Y.; Kidwai, A. R.; Indian J. Chem. 1963, 1, 99.

21. Fotie, J.; Bohle, D. S.; Olivier, M.; Gomez, M. A.; Nzimiro, S.; J. Nat. Prod. 2007, 70, 1650.

22. Desai, P. D.; Govidachari, T. R.; Nagarajan, K.; Visvanathan, N.; Indian J. Chem. 1967, 5, 41.

23. Ng, K. M.; Gray, A. I.; Waterman, P. G.; Phytochemistry 1987, 26,3251 .

Received: December 5, 2007

Web Release Date: December 12, 2008

FAPESP helped in meeting the publication costs of this article. 


\section{6-Acetonyl-N-methyl-dihydrodecarine, a New Alkaloid from Zanthoxylum riedelianum}

\section{Carromberth C. Fernandes, ${ }^{a}$ Paulo C. Vieira,${ }^{b}$ Virgínia C. da Silva, ${ }^{c}$ Evandro L. Dall'Oglio, ${ }^{c}$} Luiz E. da Silva and Paulo T. de Sousa Jr.*,

${ }^{a}$ Departamento de Ciências da Natureza, Universidade Federal do Acre, 66915-900 Rio Branco-AC, Brazil

${ }^{b}$ Departamento de Química, Centro de Ciências Exatas e de Tecnologia, Universidade Federal de São Carlos, 13565-905 São Carlos-SP, Brazil

${ }^{c}$ Departamento de Química, Instituto de Ciências Exatas e da Terra, Universidade Federal de Mato Grosso, 78060-900 Cuiabá- MT, Brazil

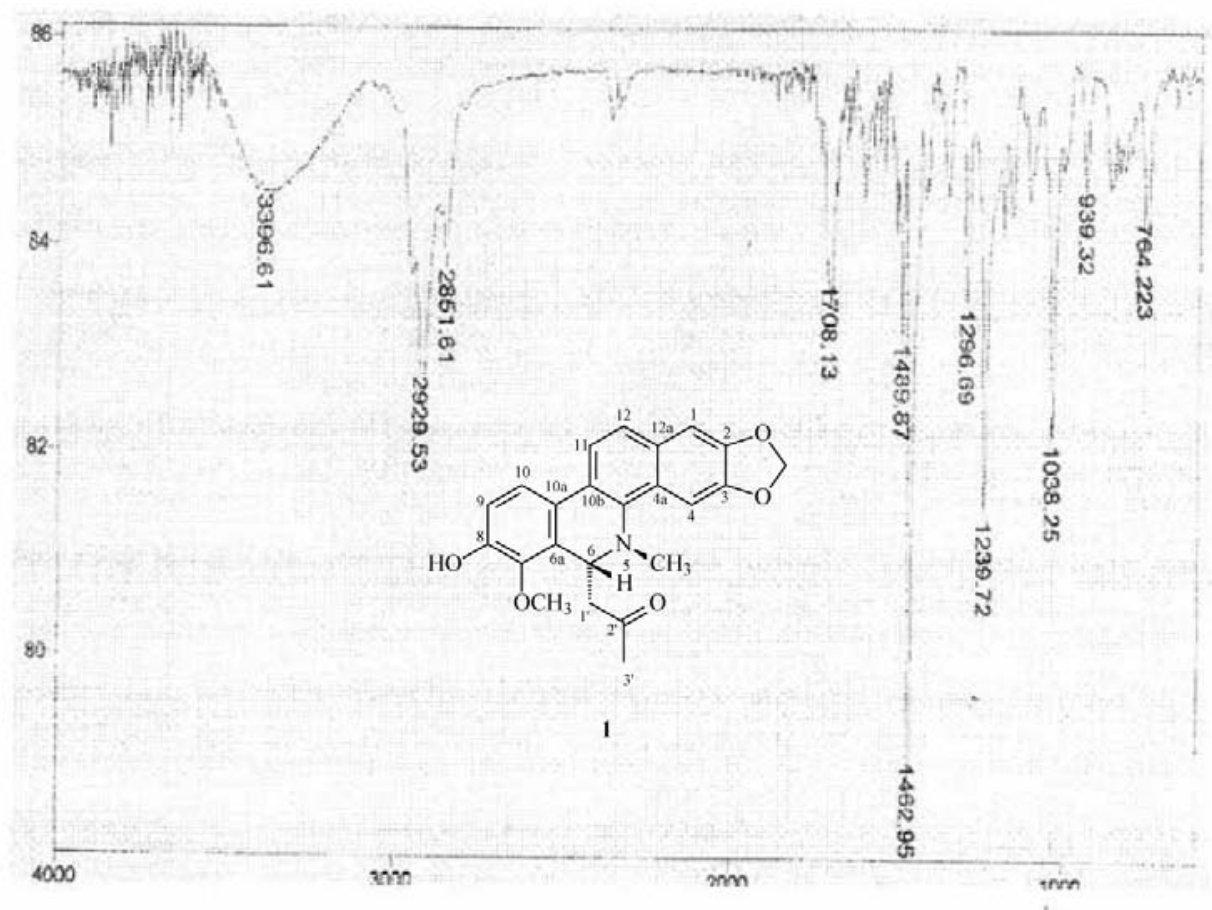

Figure S1. IR spectrum ( $\mathrm{NaCl}$ film) of $\mathbf{1}$ (6-acetonyl- $N$-methyl-dihydrodecarine).

*e-mail: teixeira@ufmt.br 

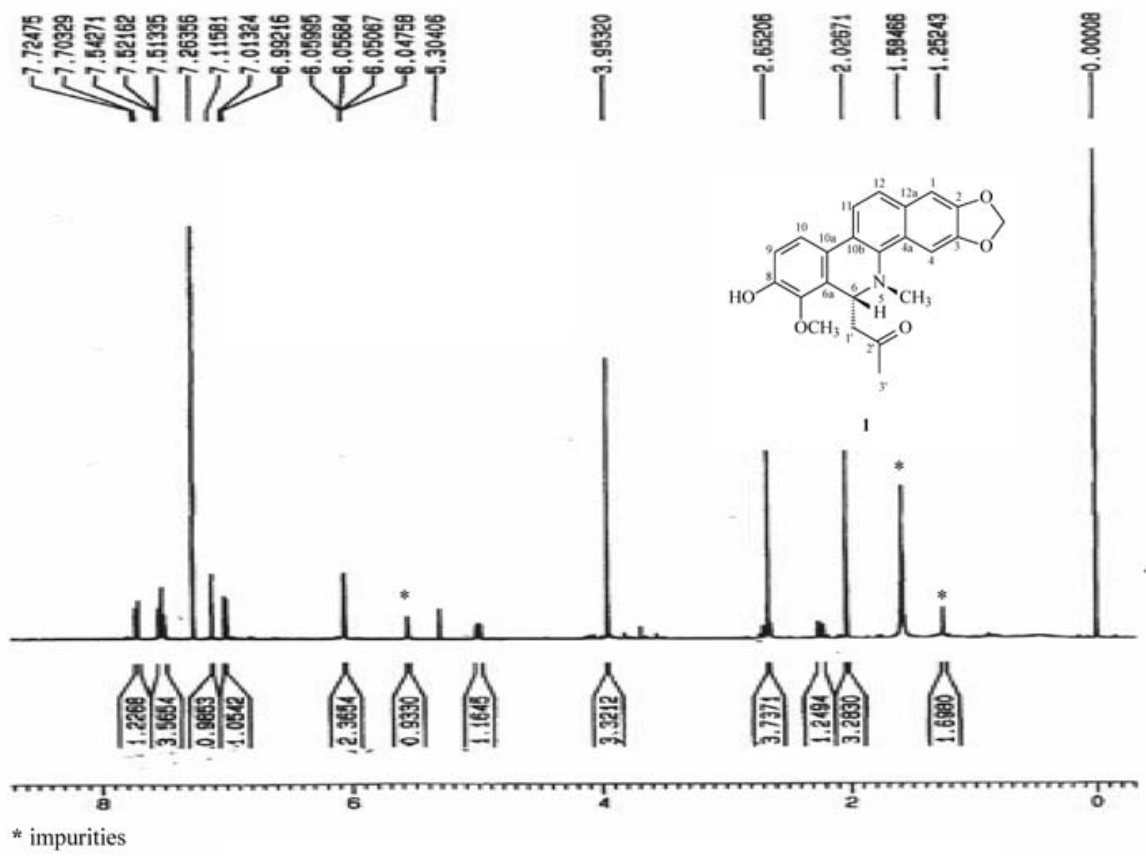

Figure S2. 'H NMR spectrum (400 $\mathrm{MHz}, \mathrm{CDCl}_{3}$ ) of $\mathbf{1}$ (6-acetonyl- $N$-methyl-dihydrodecarine).

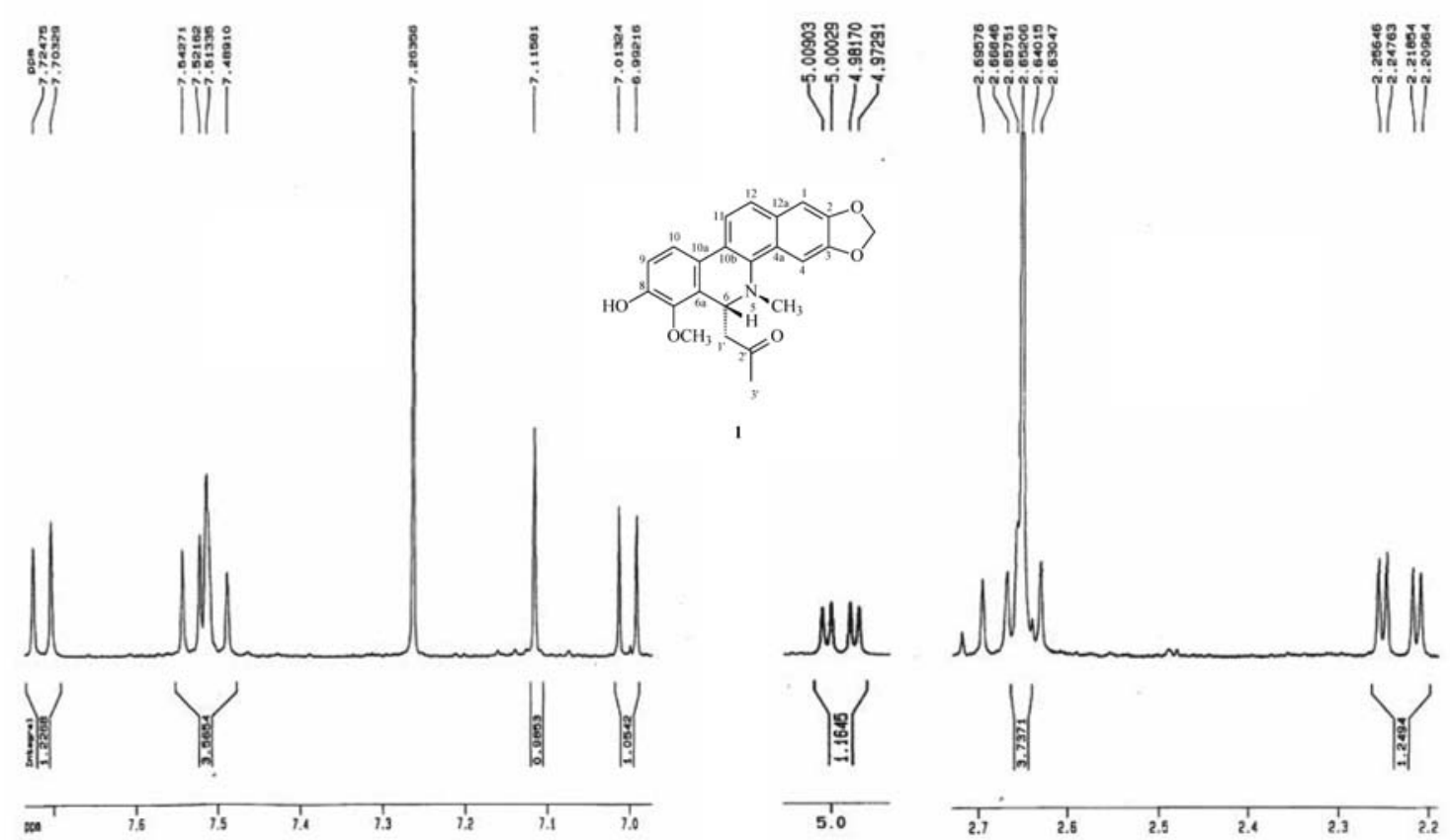

Figure S3. Expansions of the ${ }^{1} \mathrm{H}$ NMR spectrum $\left(400 \mathrm{MHz}, \mathrm{CDCl}_{3}\right.$ ) of $\mathbf{1}$ (6-acetonyl- $\mathrm{N}$-methyl-dihydrodecarine). 

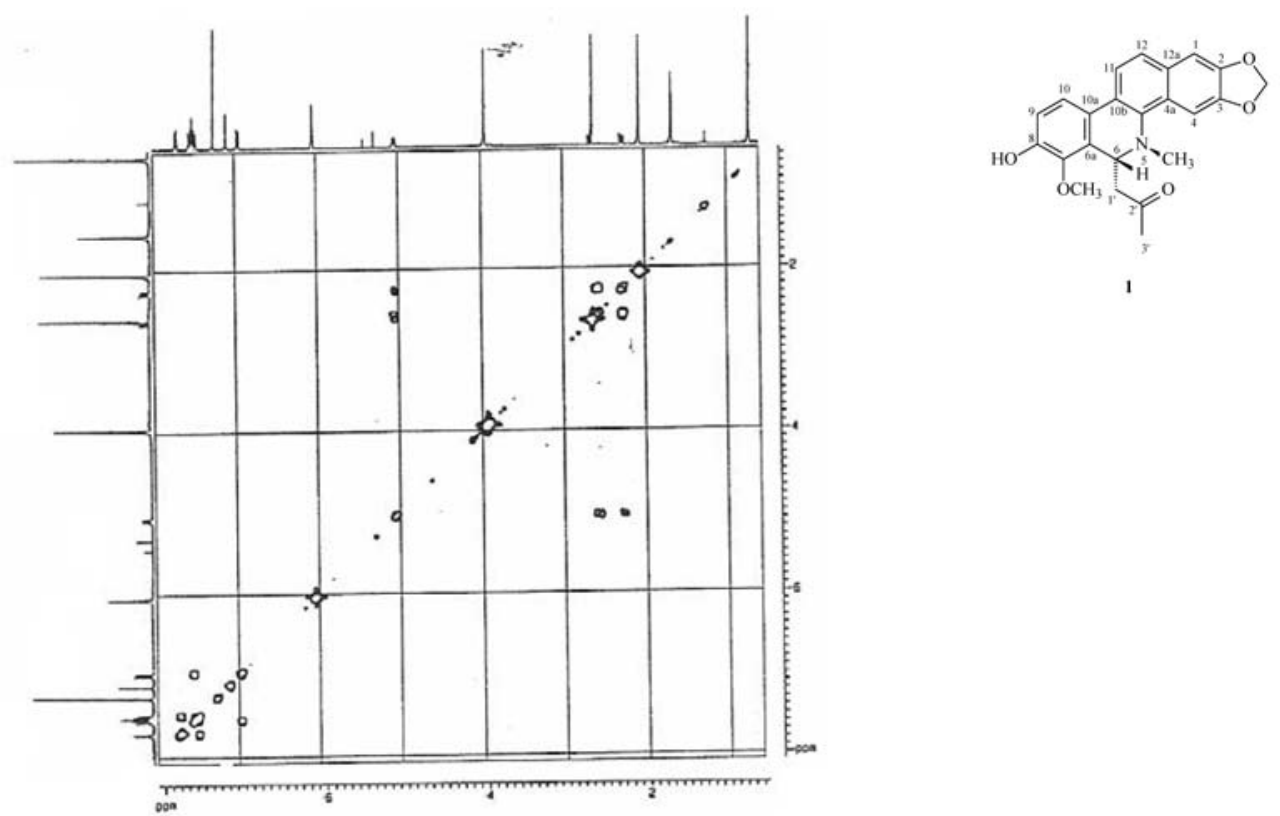

Figure S4. ${ }^{1} \mathrm{H},{ }^{1} \mathrm{H}, \mathrm{COSY}$ spectrum $\left(400 \mathrm{MHz}, \mathrm{CDCl}_{3}\right.$ ) of $\mathbf{1}$ (6-acetonyl- $\mathrm{N}$-methyl-dihydrodecarine).

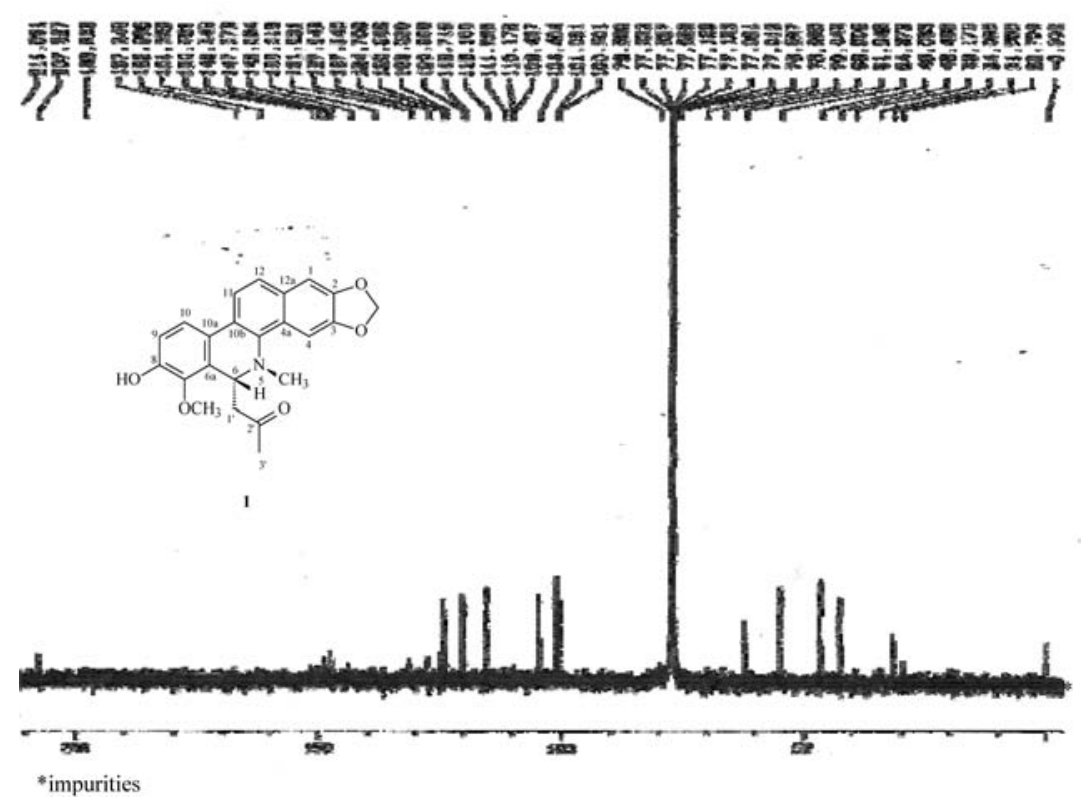

Figure S5. ${ }^{13} \mathrm{C}$ NMR spectrum (100 MHz, $\mathrm{CDCl}_{3}$ ) of $\mathbf{1}$ (6-acetonyl- $N$-methyl-dihydrodecarine). 

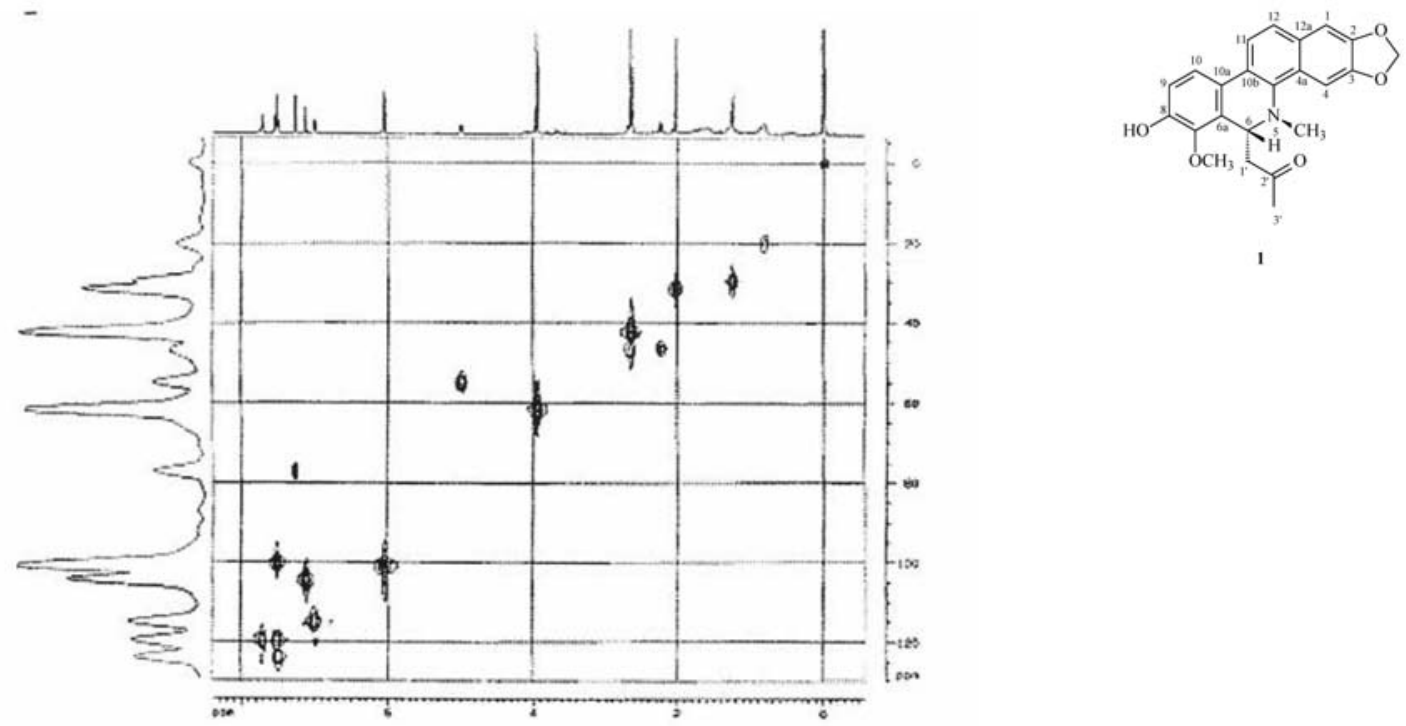

Figure S6. $g \mathrm{HSQC}$ spectrum $\left(100 \mathrm{MHz}, \mathrm{CDCl}_{3}\right.$ ) of $\mathbf{1}$ (6-acetonyl- $N$-methyl-dihydrodecarine).
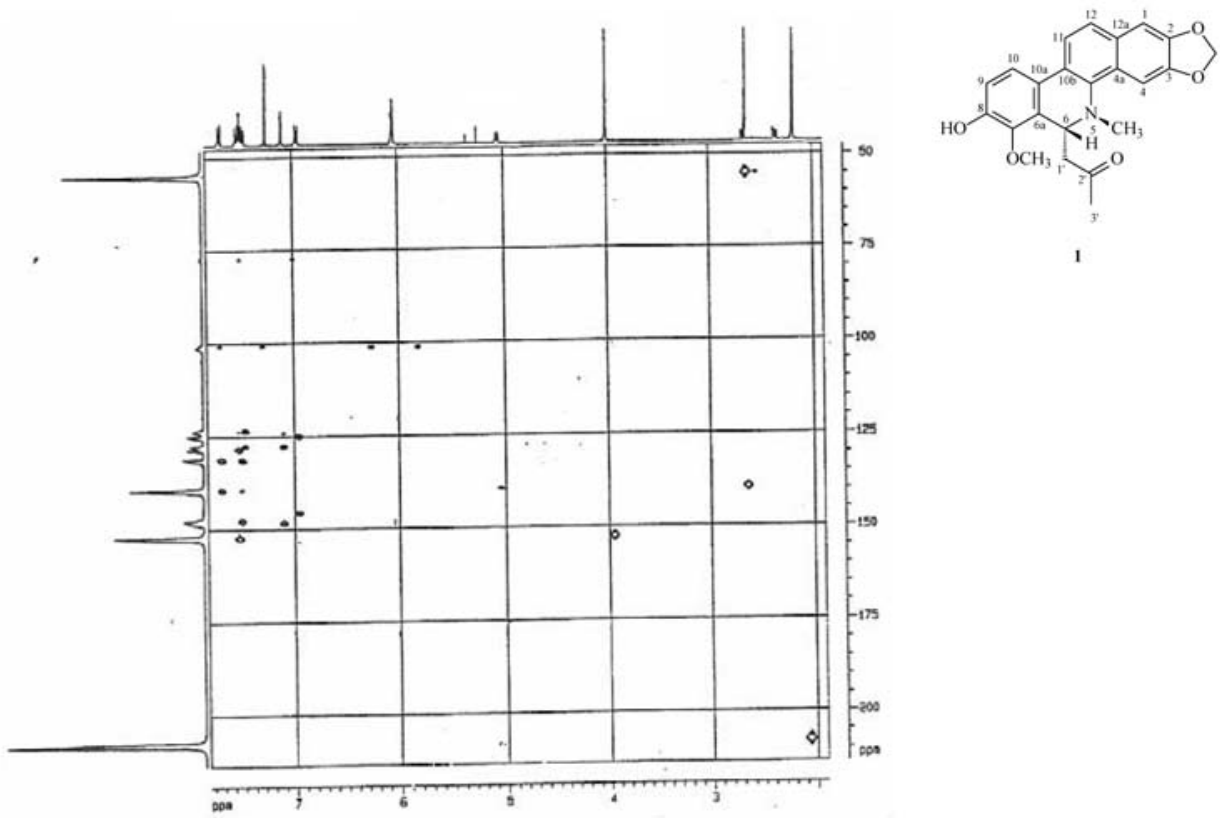

Figure S7. $\mathrm{HMBC}$ spectrum (100 MHz, $\mathrm{CDCl}_{3}$ ) of $\mathbf{1}$ (6-acetonyl- $N$-methyl-dihydrodecarine). 


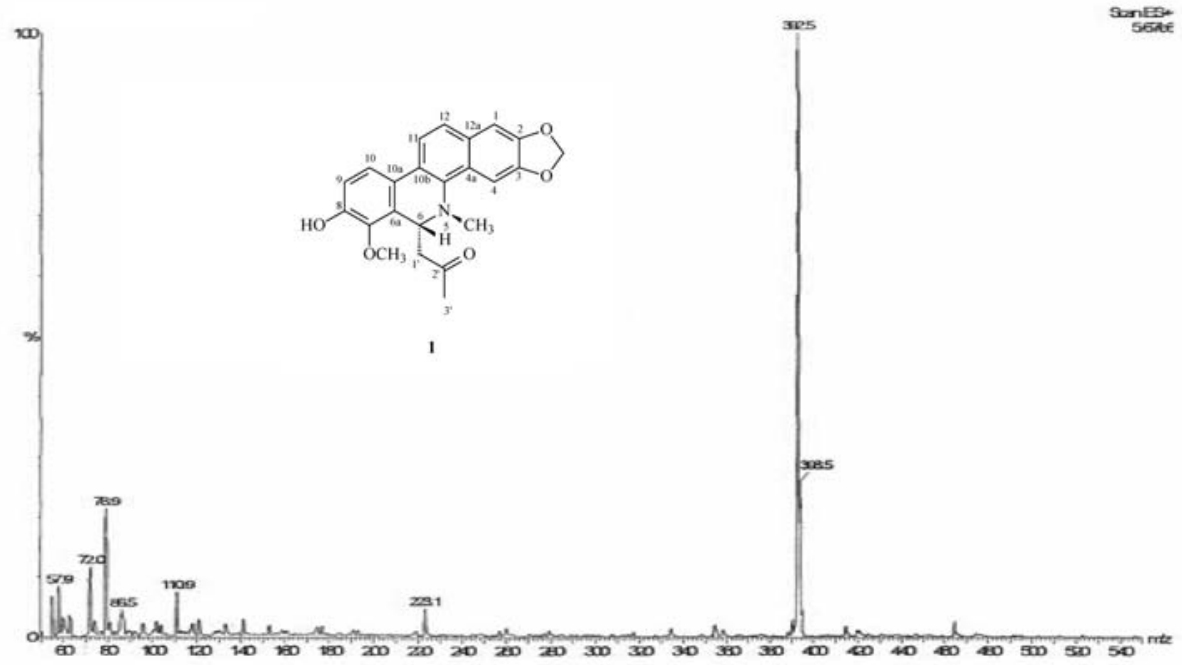

Figure S8. EIMS (+) of 1 (6-acetonyl- $N$-methyl-dihydrodecarine).

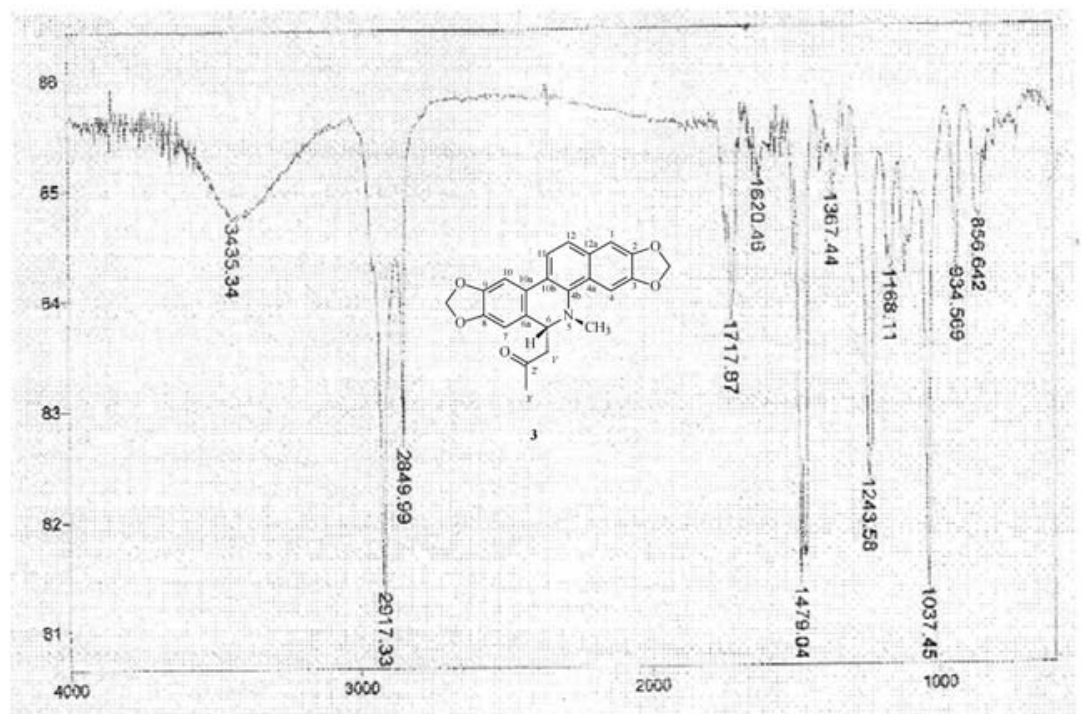

Figure S9. IR spectrum ( $\mathrm{NaCl}$ film) of $\mathbf{3}$ (6-acetonyldihydroavicine). 


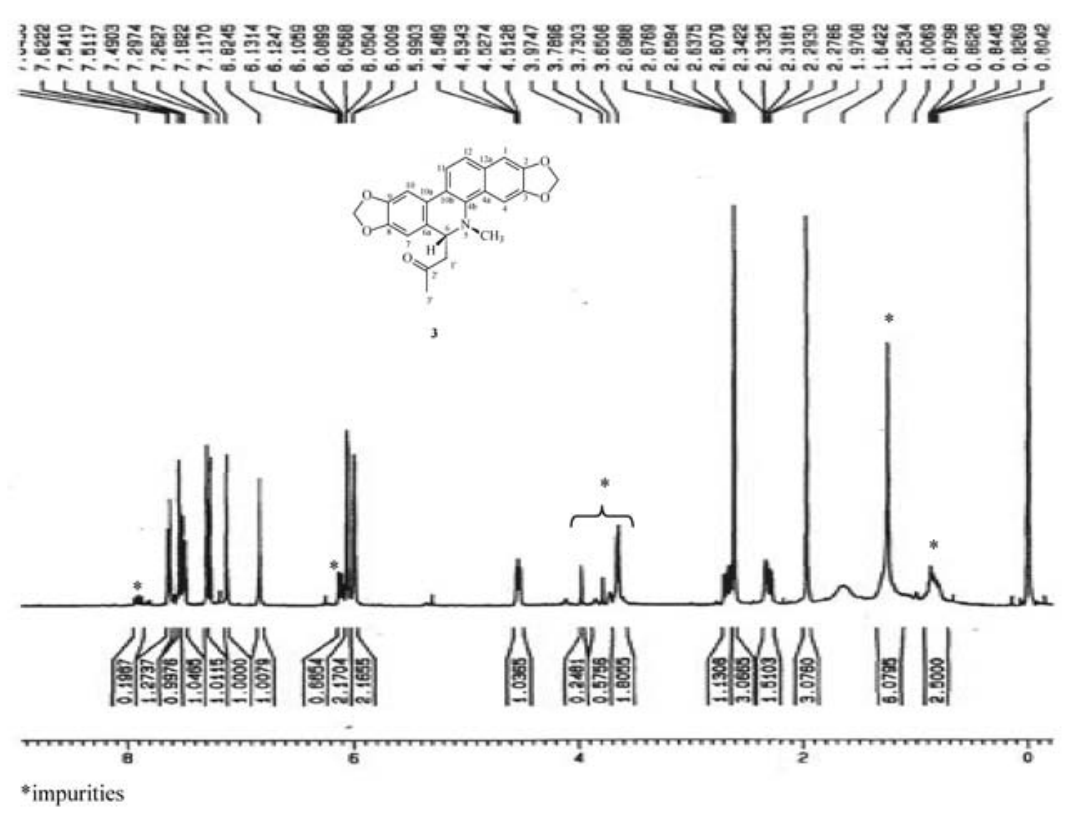

Figure S10. ${ }^{1} \mathrm{H}$ NMR spectrum (400 $\mathrm{MHz}, \mathrm{CDCl}_{3}$ ) of $\mathbf{3}$ (6-acetonyldihydroavicine).

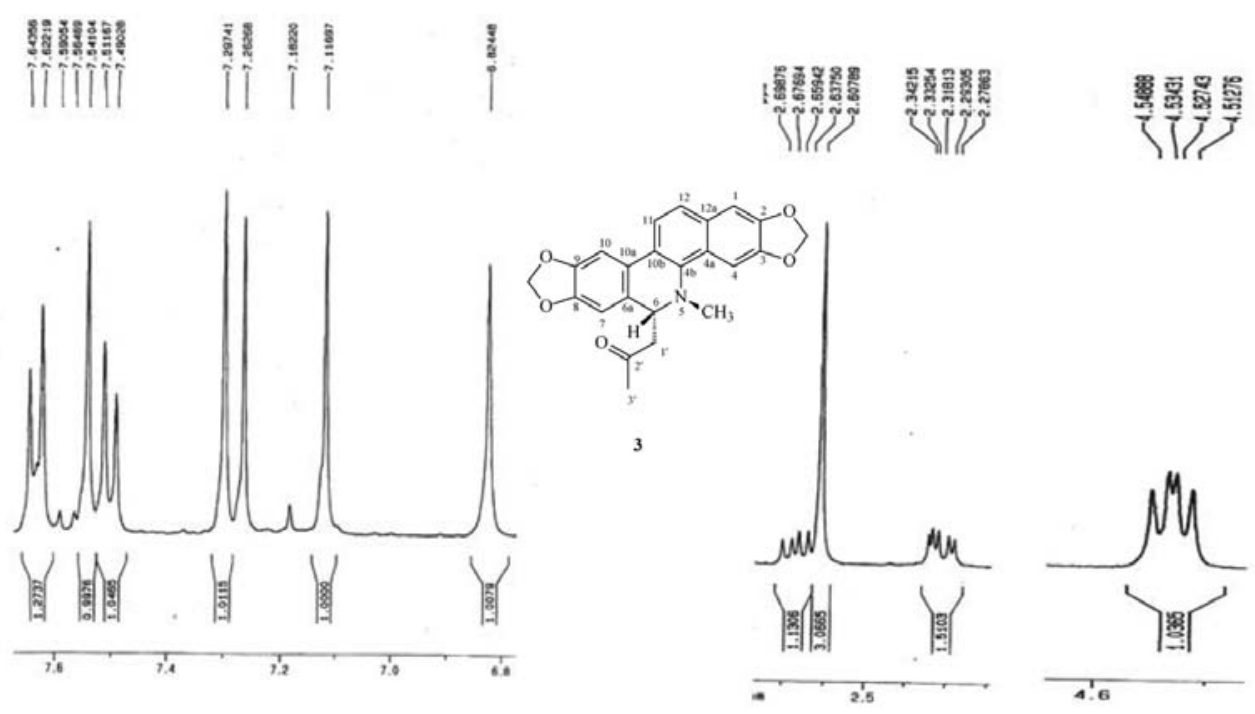

Figure S11. Expansions of the ${ }^{1} \mathrm{H}$ NMR spectrum ( $400 \mathrm{MHz}, \mathrm{CDCl}_{3}$ ) of $\mathbf{3}$ (6-acetonyldihydroavicine). 


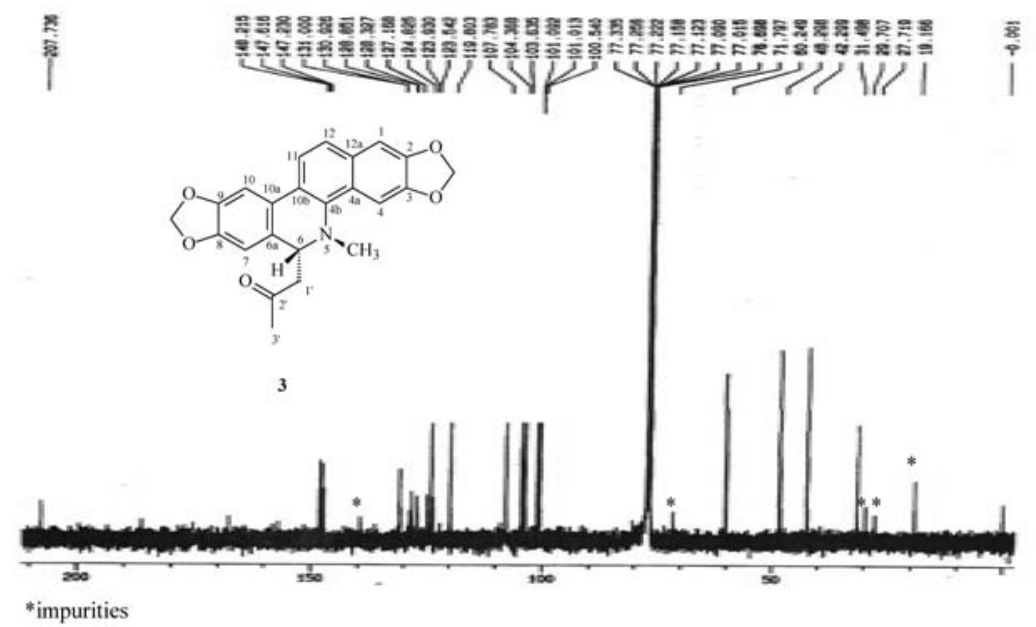

Figure S12. ${ }^{13} \mathrm{C}$ NMR spectrum (100 MHz, $\mathrm{CDCl}_{3}$ ) of $\mathbf{3}$ (6-acetonyldihydroavicine).
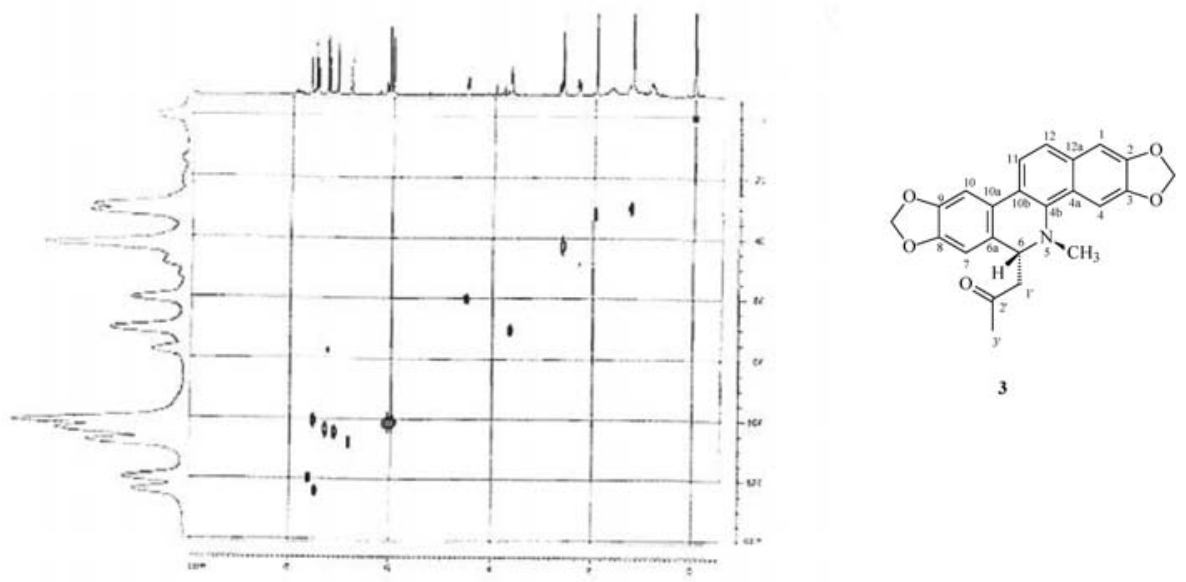

Figure S13. $g \mathrm{HSQC}$ spectrum (100 MHz, $\mathrm{CDCl}_{3}$ ) of $\mathbf{3}$ (6-acetonyldihydroavicine).

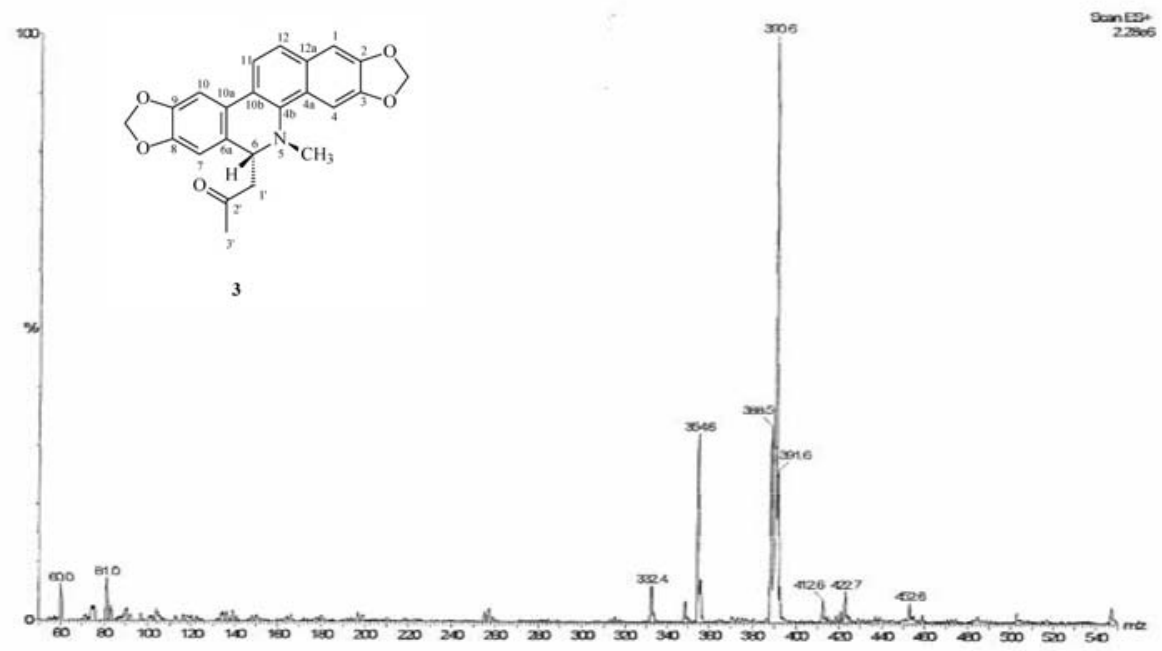

Figure S14. EIMS (+) of $\mathbf{3}$ (6-acetonyldihydroavicine). 